\title{
L'analyse du discours et les études rhétoriques
}

Discourse Analysis and Rhetorical Studies

\section{Christopher Eisenhart et Barbara Johnstone}

Traducteur : Sivan Cohen-Wiesenfeld et Ruth Amossy

\section{(2) OpenEdition \\ Journals}

Édition électronique

URL : http://journals.openedition.org/aad/1415

DOI : $10.4000 /$ aad. 1415

ISSN : 1565-8961

Éditeur

Université de Tel-Aviv

Référence électronique

Christopher Eisenhart et Barbara Johnstone, "L'analyse du discours et les études rhétoriques »,

Argumentation et Analyse du Discours [En ligne], 9 | 2012, mis en ligne le 15 octobre 2012, consulté le 23 septembre 2019. URL : http://journals.openedition.org/aad/1415 ; DOI : 10.4000/aad.1415

Ce document a été généré automatiquement le 23 septembre 2019.

\section{c) (†) $९$}

Argumentation \& analyse du discours est mis à disposition selon les termes de la licence Creative Commons Attribution - Pas d'Utilisation Commerciale - Pas de Modification 4.0 International. 


\title{
L'analyse du discours et les études rhétoriques
}

\author{
Discourse Analysis and Rhetorical Studies
}

Christopher Eisenhart et Barbara Johnstone

Traduction : Sivan Cohen-Wiesenfeld et Ruth Amossy

\section{NOTE DE L'ÉDITEUR}

Dans ce numéro qui porte essentiellement sur la relation entre l'analyse du discours et l'argumentation rhétorique, nous avons tenu à insérer d'importants extraits du texte rédigé en 2008 par deux chercheurs américains, Christopher Eisenhart et Barbara Johnstone, en introduction à un ouvrage collectif illustrant l'apport de l'analyse du discours (sur laquelle Barbara Johnstone a écrit un important livre de synthèse [Johnstone 2008]) à la rhétorique comme discipline doté d'un statut important aux USA. Le texte traduit est repris à : Johnstone, Barbara \& Christopher Eisenhart (éds). 2008. Rhetoric in Detail : Discourse analyses of rhetorical talk and text (Amsterdam \& Philadelphia : John Benjamins), 3-14.

\section{Vue d'ensemble}

1 Rhetoric in Detail rassemble douze études rédigées par des chercheurs se définissant principalement comme des rhétoriciens, qui empruntent leur cadre théorique et/ou méthodologique à l'analyse du discours linguistique. Ces travaux puisent dans un éventail de ressources offertes par l'analyse du discours, dont celles de l'analyse critique du discours (Critical Discourse Analysis ou CDA), de la sociolinguistique interactionnelle, de l'analyse des récits et de l'analyse de corpus informatisée. Ils illustrent l'utilité de l'analyse du discours dans un ensemble de « sites » rhétoriques qui comprennent les discours de la mémoire publique et de l'identité collective, la rhétorique de la science et de la technologie, l'argumentation en langage courant, le 
discours médiatique et les études sur l'immigration. La méthode que ces projets partagent est ancrée dans l'attention méticuleuse portée aux détails linguistiques des discours sélectionnés, qu'il s'agisse de textes écrits ou de transcriptions de l'oral. Les auteurs adoptent une approche essentiellement qualitative et interprétative, mais celle-ci diffère de la voie qu'empruntent souvent les études rhétoriques en ce qu'elle est orientée vers les corpus plutôt que vers la théorie. Travaillant à partir de textes et de discours oraux particuliers en situation plutôt que de modèles abstraits, ils choisissent des approches qui explorent de façon systématique les raisons pour lesquelles des énoncés particuliers revêtent la forme particulière qui est la leur. Plutôt que de partir de la théorie en cherchant à l'étayer, cette approche prend en compte, d'entrée de jeu, les multiples sources de contraintes textuelles. Si les études qui composent les différents chapitres traitent de questions rhétoriques de façons variées, elles n'en partagent pas moins trois caractéristiques d'ordre méthodologique. Elles sont empiriques, dans le sens où elles sont fondées sur l'observation plutôt que sur la seule introspection; elles sont ethnographiques en ce qu'elles cherchent à comprendre les fonctionnements rhétoriques du discours et de son contexte à travers les yeux et l'esprit de ceux qui les manient; et elles sont étayées, retournant sans cesse aux données afin de construire la théorie qui doit en rendre compte.

Issues d'une heuristique analytique plutôt que d'un cadre théorique préexistant, ces études montrent le potentiel qu'offre aux études de rhétorique et à la critique une théorie fondée sur le discours et caractérisée par l'observation. Dans la mesure où la rhétorique élargit ses centres d'intérêt du programmé au spontané et du public au privé, les rhétoriciens reconnaissent le besoin de trouver de nouvelles méthodes [...] L'analyse du discours peut à son tour découvrir de nouveaux instruments. Les premiers théoriciens du discours dans la tradition intellectuelle gréco-romaine étaient les philosophes et les sophistes qui décrivaient et enseignaient la parole publique aux citoyens dont la voix avait un impact dans la nouvelle Athènes démocratique du $5^{\mathrm{e}}$ siècle av. J-C; les auteurs de cet ouvrage viennent revigorer cette tradition, plus particulièrement en Amérique du Nord, au sein de la «nouvelle rhétorique » du $20^{\mathrm{e}}$ siècle. Selon des modalités neuves, plusieurs de ces études empruntent les instruments analytiques traditionnels de la rhétorique - figures du discours, topoï, lignes d'arguments, invention et style, ethos, logos et pathos - montrant comment ils peuvent nourrir et être nourris par l'attention que portent les analystes du discours à la façon dont le lexique et la syntaxe peuvent évoquer des styles, des genres, des textes et locuteurs préalables, créant par là même des relations sociales et des mondes expérimentaux dans le discours oral et écrit.

\section{Méthodes et problématiques dans les études de rhétorique nord-américaines}

3 Les rhétoriciens ont toujours adopté une approche inclusive et accueillante en matière de méthode analytique. En plus de l'usage qu'ils font du vocabulaire analytique de la rhétorique classique, les praticiens ont emprunté et adapté des méthodes provenant d'autres disciplines, reprenant par exemple à la philosophie le raisonnement fondé sur l'intuition, et à la théorie littéraire et sociologique les techniques de l'« explication de textes » et une variété de perspectives critiques et théoriques. Ces instruments ont été développés pour répondre aux questions que soulevaient les genres soigneusement 
programmés, et souvent institutionnels, qui constituaient l'objet principal de la critique rhétorique. Cependant, les centres d'intérêt des rhétoriciens s'élargissent désormais de la sphère publique à la sphère privée, de la rhétorique officielle à la rhétorique vernaculaire, de l'art oratoire aux discours écrits et aux multimédias, et des discours soigneusement travaillés à celui, spontané, qui émerge des situations rhétoriques fugaces de la vie quotidienne. Aujourd'hui on s'interroge, non seulement sur la rhétorique politique, mais aussi sur celle de l'histoire et de la culture populaire ; non seulement sur la rhétorique de la sphère publique mais aussi sur celle de la rue, du salon de coiffure ou de l'internet; non seulement sur la rhétoricité de l'argument formel mais aussi sur celle de l'identité personnelle. Pour traiter de ces nouvelles questions et de ces "sites", il importe d'ajouter aux méthodes traditionnelles de nouvelles techniques d'analyse du langage des textes et des discours oraux, ainsi que des modalités de description des contextes socioculturels et matériels du discours [...].

\section{Contexte et agentivité}

4 Parmi les pratiques les plus fondamentales de la rhétorique, on compte l'étude du discours en contexte. Dans son traité de rhétorique, Aristote discutait des composantes de la situation de discours et établissait très clairement le rapport de la rhétorique au discours public et civique. La restriction au discours civique que s'était imposée la rhétorique n'est plus de mise, mais la prémisse centrale selon laquelle le discours doit être modelé par le contexte est toujours d'actualité.

5 Tout aussi fondamental est l'intérêt de la rhétorique envers le pouvoir et les choix que le rhéteur - orateur ou scripteur - exerce dans une situation donnée. Certaines définitions du discours rhétorique voient sa spécificité dans le fait qu'il constitue un discours destiné à effectuer un changement dans la situation pour laquelle il a été forgé, et doté de la capacité de le faire. Il en résulte que l'agentivité est un trait essentiel des espaces au sein desquels sont traités des problèmes rhétoriques. Leff, par exemple, discute de l'agentivité comme d'une source de tension entre les conceptions du moi des Lumières et les critiques post-modernes de ces conceptualisations, dans une tradition sophistique et cicéronienne qu'il rebaptise « rhétorique humaniste » :

l'approche humaniste implique une conception de l'agentivité dont l'ambiguïté est féconde, et qui positionne l'orateur à la fois comme individu guidant un auditoire, et comme membre d'une communauté façonné et contraint par les exigences de l'auditoire. [Cette tradition peut comprendre] une certaine méfiance à l'égard de la théorie abstraite non seulement dans le domaine de la rhétorique, mais aussi dans celui de l'éthique et du politique ; la conviction que le discours, particulièrement celui qui permet le débat contradictoire, joue un rôle fondamental dans la vie publique ; une valorisation et une idéalisation de l'éloquence qui implique un lien étroit avec la vertu ; et une conception de celle-ci qui la relie résolument à l'activité politique (Leff 2011 [2003], 1 et 2).

6 La tentative de définir et d'étudier les espaces rhétoriques et les tensions entre l'agentivité rhétorique et les contraintes qu'on reconnaît peser sur le texte, s'est avérée l'un des problèmes théoriques les plus féconds de la rhétorique contemporaine (cf. Bitzer 1968 ; Vatz 1973). Et c'est en relation avec cet espace de questionnement que les études rhétoriques ont relevé le défi de rendre compte de l'interaction entre l'agentivité rhétorique et le contexte. Durant ces dernières décennies, un travail inspiré de la théorie de la sphère publique d'Habermas (1989) a permis de penser cette 
intersection de façon fructueuse. L'un des soucis majeurs des études rhétoriques est l'étude du discours concret d'un agent dans la sphère publique. Comme l'écrit Hauser,

Au mieux, la rhétorique de la démocratie est impure selon les critères de la raison posés par les Lumières. En conséquence, certains penseurs comme Habermas, qui a été l'un des plus grands champions d'une conception du discours comme point d'ancrage conceptuel de la démocratie, ont considéré que la prédilection de la rhétorique pour la stratégie était problématique. Mais exclure les processus rhétoriques de notre appréciation des débats courants de la démocratie exclut en même temps l'agentivité qui permet de prendre des décisions démocratiques. Avant de pouvoir réhabiliter la vie publique, nous devons comprendre comment les discours qui s'y déroulent concrètement la modèlent. Sinon, toute critique ou remède mis en avant reste purement analytique, produisant des conclusions issues d'hypothèses posées a priori sur ce que sont les standards rationnels/idéologiques d'un accord "valide », mais qui manquent d'un référent empirique sous la forme de la méthode discursive réelle à laquelle les membres de la sphère publique ont recours (Hauser 1999 : 273).

7 Le programme de Hauser consiste dès lors à adopter une attitude empirique dans l'étude de la façon dont les rhéteurs agissent dans l'espace public, valorisant l'étude des discours vernaculaires aux dépens des généralisations théoriques fondées sur une lecture solitaire des discours institutionnels. Ceci entre en résonance avec le conseil d'Asen (2004) suggérant de développer un sens de la citoyenneté démocratique en passant de «ce qui constitue la citoyenneté à la question de savoir comment la citoyenneté procède », et avec le conseil de Simons (2000:448-9) de "procéder à partir du particulier, du local, de l'unique - vers une théorie de l'événement spécifique - à partir de laquelle on pourrait dériver un sens des limites, possibilités et compromis mis en jeu par la sélection de cette option plutôt que d'une autre ». Illustrant cette quête, Johnstone (1996) utilise l'analyse du discours pour investiguer la façon dont le complexe de l'agentivité rhétorique et du contexte se constitue dans le discours, et McCormick (2003) se concentre sur les analyses rhétoriques des discours vernaculaires susceptibles de bénéficier de l'apport de la CDA.

$[\ldots]$

\section{Qu'est-ce que l'analyse du discours?}

8 Les linguistes qui se veulent des spécialistes d'analyse du discours examinent ce que l'étude de l'utilisation du langage peut nous apprendre sur le langage lui-même et sur les locuteurs. A l'encontre des linguistes de la tradition chomskyenne, ils analysent des textes écrits, ou des transcriptions de discours oraux ou en langage des signes, plutôt que de se fonder sur leurs propres intuitions grammaticales. Ils s'intéressent à la structure et à la fonction de fragments de discours ou de textes au-delà de l'unité de la phrase; ils examinent comment la structure des phrases est influencée par leur fonctionnement dans les contextes linguistiques et sociaux dans lesquels elles se déploient. Par « discours » ils entendent des exemples réels de conversation, d'écrits ou de communication linguistique dans d'autres media. Certains d'entre eux tentent explicitement de relier les caractéristiques du discours pris dans ce sens avec des aspects de ce que les chercheurs de la tradition foucaldienne appellent le « discours »: des corpus d'idées et de pratiques sociales en circulation pouvant inclure des façons de parler. D'autres ont des objectifs différents. Certains s'intéressent aux types de questions auxquelles les linguistes se sont toujours confrontés : comment le langage 
est-il représenté dans l'esprit, quelle est la meilleure manière de modéliser la production et l'interprétation du discours, comment le langage s'acquiert, change, etc. D'autres explorent les liens entre les phénomènes discursifs et sociaux dans une grande variété de contextes, comprenant la communication institutionnelle, la construction discursive de l'identité et de la mémoire, le discours politique, le comportement organisationnel, la communication au sein de la famille etc.

Comme d'autres courants de la linguistique contemporaine, l'analyse du discours puise ses racines historiques dans la philologie du dix-neuvième siècle, c'est-à-dire dans une étude diachronique (historique) du langage qui a comme objet l'exégèse des textes. A la suite de l'appel de Ferdinand de Saussure (1916) à recentrer l'étude du langage sur la structure synchronique, les approches dominantes de la plus grande partie du vingtième siècle se sont attachées aux sons, aux phrases et aux propositions plutôt qu'au discours suivi. Au début des années 1960, cependant, des linguistes de diverses traditions intellectuelles commencèrent à converger vers deux idées-forces sur le discours reliées entre elles : 1) l'idée selon laquelle la structure des expressions et des phrases est déterminée en partie par la manière dont elles fonctionnent dans les conversations et dans les textes, et 2) l'idée que les textes et les conversations sont modelés, tout comme les phrases, sur des modèles de structure reproductibles qui pourraient être considérés comme une "grammaire". En Grande-Bretagne, M. A. K. Halliday, à partir du travail de J. R. Firth, commença à développer une "grammaire systémique fonctionnelle » et à s'interroger sur la cohésion des phrases à l'intérieur des textes (Halliday 1994 ; Eggins 1994). Aux Etats-Unis, Kenneth Pike et d'autres linguistes associés au Summer Institute of Linguistics développèrent une méthode similaire de compréhension de la structure de la phrase et du discours basée sur la fonction, qu'ils appelèrent la grammaire tagmémique (Pike 1967). En même temps, l'émergence de la sociolinguistique variationniste, de l'analyse conversationnelle, de la sociolinguistique interactionnelle et de l'ethnographie de la communication, mit le discours à la portée des étudiants dans les domaines respectifs de l'évolution du langage, de la sociologie du langage et de la linguistique anthropologique (Labov 1963, 1972; Sacks, Schlegoff et Jefferson, 1974 ; Ten Have 1999 ; Gumperz et Hymes 1972).

En France, les linguistes marxistes commencèrent à explorer la manière dont l'idéologie est construite et révélée par le discours (Pêcheux 1969). Un peu plus tard, les linguistes influencés par la théorie sociale de l'école de Birmingham portèrent à l'attention des anglophones une approche critique similaire du discours, suggérant que, puisque l'analyse du discours ne peut jamais être purement descriptive, son but devait être de révéler comment le pouvoir circule, généralement de manière invisible, à travers le discours (Fairclough 1992, 2003 ; Wodak 1996, 2005). Cette approche, la Critical Discourse Analysis, garde une grande influence [...]. Les autres travaux en analyse du discours sont plus éclectiques, et font appel à la pragmatique, à la sociolinguistique, à la linguistique interactionnelle et aux divers courants de la théorie de l'argumentation, de la rhétorique, de la sociologie, de la littérature et de l'anthropologie. De nombreux analystes, en particulier ceux dont la discipline d'origine est la linguistique, continuent d'être intéressés essentiellement par des questions de langue, mais l'utilisation de l'analyse du discours, quelle qu'en soit sa définition, comme méthode d'analyse systématique et approfondie est devenue de plus en plus interdisciplinaire. Les manuels ne partent plus du présupposé selon lequel tous les spécialistes d'analyse du discours sont des linguistes; une formation en analyse du 
discours est parfois proposée dans le contexte de programmes "d'études du discours » dans le cadre de diverses spécialisations universitaires; et des périodiques comme Discourse Studies, Discourse in Society, Disourse and Communication et Text and Talk publient les travaux de chercheurs relevant de filiations disciplinaires variées.

\section{Une approche heuristique du discours}

11 Les analyses du discours illustrées ici prennent toutes pour point de départ le travail d'Alaton L. Becker (1995; voir également Johstone 2008). Nous partons d'une technique heuristique, d'une approche particulariste, interprétative mais systématique qui a pour but de découvrir pourquoi un texte donné est tel qu'il est. Les spécialistes d'analyse du discours travaillent à partir de types de matériaux variés, y compris des transcriptions d'interactions enregistrées sur des supports audio ou vidéo, des documents écrits, des textes transmis par la tradition orale comme des proverbes, ou des reproductions de communication en ligne. Leur matériel est parfois uniquement verbal et parfois comprend des photos, des gestes, des regards et d'autres modalités. Mais quel que soit le type de discours considéré, la question de base posée par l'analyste est toujours: "Pourquoi ce segment de discours est-il tel qu'il est? Pourquoi ne peut-il être différent ? Pourquoi ces mots particuliers, dans cet ordre particulier?»

12 Pour répondre à ces questions, nous devons évidemment réfléchir à ce sur quoi porte le « texte », car il est clair que ce dont une personne parle a un impact sur ce qu'elle dit et sur la manière dont elle le dit. Nous devons également prendre en considération la personne qui parle, qui écrit ou qui signe, et celle qui est considérée, dans un contexte socioculturel particulier, comme responsable de ce qui est dit; qui était le public visé ainsi que les auditeurs ou lecteurs réels, car il est clair que les participants à une situation et la manière dont leur rôle est défini influencent ce qui est dit et la manière dont c'est dit. Nous devons penser à ce qui a motivé le texte, dans quelle mesure il est adapté à l'ensemble de ce que les gens font conventionnellement avec le discours dans un tel contexte, et dans quelle mesure son canal ou ses canaux de production influencent sa forme. Nous devons réfléchir sur le langage employé, sur le comportement que ce langage encourage les orateurs et les scripteurs à adopter, et sur ce qu'il est relativement difficile de faire avec un tel langage. Nous devons réfléchir également à la structure du texte et à la manière dont il s'intègre dans des structures plus larges d'ensembles de textes ou d'interactions.

On peut répartir les questions à poser sur un texte en six grandes catégories. Chacune d'entre elles correspond à une des manières dont les contextes modèlent les textes, et inversement. Chacun de ces aspects de la construction du texte est à la fois une source de contrainte - une raison qui fait que les textes sont d'un certain type et pas d'un autre - et une source de créativité, dans la mesure où les locuteurs, les signataires et les scripteurs s'expriment en manipulant les modèles devenus conventionnels.

- Le discours est modelé par le monde, et modèle le monde.

- Le discours est modelé par le langage, et façonne le langage.

- Le discours est modelé par les participants, et façonne les participants.

- Le discours est modelé par le discours antérieur, et modèle les possibilités de discours futur.

- Le discours est modelé par son moyen de diffusion, et en transforme les possibilités.

- Le discours est modelé par son but, et il modèle des buts possibles. 

systématique des éléments potentiellement intéressants et importants pour l'étude d'un texte ou d'un ensemble de textes. Une "heuristique» est un ensemble de procédures de découverte à appliquer méthodiquement, ou un ensemble de thèmes à prendre en considération systématiquement. Contrairement à des instructions, les procédures d'une heuristique n'ont pas besoin d'être suivis dans un ordre particulier, et il n'existe pas de manière fixe de les suivre. Une heuristique n'est pas une suite mécanique d'étapes, et rien ne garantit que son utilisation conduise à une explication définitive unique. Une bonne heuristique fait appel à de multiples théories plutôt qu'à une seule. Celle que nous utilisons ici nous oblige à penser, par exemple, à la manière dont le discours est modelé par les idéologies qui font circuler le pouvoir dans la société, mais nous enjoint également à penser à la façon dont le discours est modelé par la mémoire de discours antérieurs, ainsi que par d'autres sources de créativité ou de contrainte. Il est possible que nous finissions par décider, pour chaque cas particulier, que l'approche la plus utile est celle qui permet de voir comment le pouvoir circule dans le discours, ou celle qui nous aide à décrire "l'intertextualité ", ou à découvrir les relations entre le texte et son support, le langage utilisé, ou encore les buts de ses producteurs ou leurs relations sociales. L'heuristique est un premier pas qui aide l'analyste à discerner quelles sont les théories nécessaires pour relier les observations particulières sur le discours faites dans le cours de sa démarche, à des affirmations générales sur le langage, la vie humaine ou la société. C'est une manière d'ancrer l'analyse dans le discours, plutôt que de partir d'une théorie choisie d'avance et d'utiliser des textes pour la mettre à l'épreuve ou l'illustrer.

\section{Situer l'heuristique dans la théorie rhétorique}

Chacun des six éléments de notre analyse heuristique fait appel à un courant de pensée sur le langage et la communication qui est, du moins en partie, déjà familier aux rhétoriciens. L'idée selon laquelle les textes et leur interprétation sont à la fois forgés par le monde et le forgent à leur tour est enracinée dans la théorie rhétorique et linguistique sur le rôle de la référence dans la production et l'interprétation du discours. Le discours émerge du ou des univers qui sont présumés exister en dehors de lui, l'univers des producteurs et interprétateurs de textes. Le fait qu'un discours soit considéré comme portant ou non sur quelque chose influe sur la manière dont il est interprété. Un discours qui est considéré comme ne se référant à rien est susceptible d'être considéré comme insensé ou fou; il peut être le résultat d'une expérience linguistique comme le dadaïsme en poésie, ou bien être requis par un rituel. La tradition de la pensée occidentale sur le langage a eu tendance à privilégier le discours référentiel et à imaginer que le discours reflète (au moins idéalement) le monde préexistant. Mais, comme nous l'ont continuellement montré les philosophes (Foucault 1980), les rhétoriciens (Burke 1945) et les linguistes (Sapir 1949; Whorf 1941) du 20e siècle, l'inverse est également vrai, et peut-être plus vrai encore : l'univers humain est façonné par le discours.

Lorsque nous signalons la manière dont les textes et leurs interprétations sont modelés par les ressources structurelles disponibles, nous alléguons le fait (bien connu des rhétoriciens qui s'intéressent au style et à la disposition) qu'il existe des moyens conventionnels de structurer des textes à tous les niveaux. Parler une langue, comme 
l'anglais ou le coréen, signifie utiliser des moyens conventionnels de structurer des syllabes (un nouveau mot anglais peut commencer par la syllabe pri mais pas par ngi), des mots (le s qui marque le pluriel d'un mot en anglais se place après la racine, pas avant), des phrases (dans les phrases affirmatives en anglais le sujet précède généralement le verbe). Il existe de même des manières conventionnelles d'organiser les unités de discours plus grandes, certaines étant spécifiques d'une culture et d'autres le résultat de processus de cognition humains. Ceci inclut des façons de passer de l'information familière à l'information nouvelle, par exemple, de l'exemple à la généralité ou inversement, ou bien des questions aux réponses.

17 L'affirmation selon laquelle le discours est façonné par les relations interpersonnelles des participants et aide inversement ces relations à s'organiser, doit évoquer les conceptions traditionnelles de l'auditoire et de l'ethos rhétorique, ou les nouvelles façons de penser la manière dont la position et le rôle du locuteur se modèlent et s'autorisent mutuellement, dans le contexte de structures de pouvoir plus larges. Les relations interpersonnelles liées au discours comprennent les relations entre les locuteurs et les scripteurs, les auditoires et les allocutaires tiers représentés dans les textes, ou impliqués dans leur production et leur interprétation.

Une autre observation de l'heuristique, selon laquelle le discours est façonné par les attentes créées par le discours familier et les nouvelles instances de discours nous aident à constituer nos attentes concernant le discours futur, devrait également être familière aux rhétoriciens pratiquant les théories contemporaines sur le genre (Miller 1984 ; Swales 1990) et l'intertextualité (Bakhtine 1986). Les relations intertextuelles nous permettent d'interpréter les nouvelles instances de discours en référence aux activités et aux catégories stylistique et formelles familières. Les utilisations du discours sont aussi variées que les cultures humaines, mais les activités récurrentes relatives au discours engendrent des processus relativement fixes, qui incluent souvent des façons de parler et des types de textes eux aussi fixes et passés à l'état de routine.

Les spécialistes de rhétorique visuelle (Handa 2004 ; Prelli 2006) et ceux intéressés par la multimodalité (Hodge et Kress 1988 ; Levine et Scollon 2004 ; Scollon et Scollon 2003) devraient également être ouverts à l'idée selon laquelle le discours est tributaire des limites et des possibilités offertes par les supports médiatiques qu'il emprunte, et inversement les capacités de ces derniers sont forgées par leur utilisation par le discours. Finalement, l'observation selon laquelle le discours est façonné par son but est à la racine de la discipline de la rhétorique et, inversement, le fait que le discours puisse élaborer des buts possibles doit recevoir un écho chez tous ceux qui se penchent sur le fonctionnement de la rhétorique épidictique et délibérative dans des contextes contemporains.

20 Commencer à interroger les textes par tous les moyens suggérés par l'heuristique signifie que l'analyse part des textes et d'une réflexion systématique sur les contextes pertinents possibles. Le résultat en est une description large, multidimensionnelle et dense (Geertz 1983). Ceci fait, le chercheur est en mesure de se concentrer sur une ou deux questions, adoptant l'une des diverses approches possibles pour étoffer les détails. Nous accordons une attention systématique à la manière dont les textes et les discours sont produits et prennent forme, ainsi qu'aux caractéristiques structurelles et sémantiques des exemples particuliers de textes et de discours. 


\section{L'analyse du discours dans la recherche contemporaine en rhétorique}

21 Si de nombreux critiques rhétoriques ne sont guère familiers de l'analyse du discours, ils côtoient des collègues qui en ont l'expérience. Les spécialistes de rhétorique et de composition des départements d'Anglais ont longtemps considéré la linguistique comme une source d'idées et de méthodes (voir Cooper et Greenbaum 1986; Raskin et Weiser 1987). Les spécialistes de composition Barton et Stygall notent que «l'analyse du discours est la base des études de composition : chaque étude dans le champ est basé implicitement ou explicitement sur l'analyse de textes et/ou de discours dans divers contextes» (Barton et Stygall 2002:1). L'ouvrage de Barton et Stygall rassemble des travaux de linguistes et de rhétoriciens (ou de chercheurs qui travaillent dans les deux domaines à la fois) ayant un rapport avec l'écriture en général, l'écriture universitaire, l'écriture d'une seconde langue, et le discours scientifique et professionnel, de même que l'analyse du discours dans des cours de composition. Le livre de Bazerman et Prior (2004) se concentre sur les méthodes d'étude de l'écriture, y compris l'analyse du discours linguistique. Les étudiants intéressés par l'analyse de texte quantitative peuvent se servir du manuel de Geisler; Kaufer et ses collègues (Kaufer et Butler 2000 ; Kaufer et al. 2004) ont quant à eux développé un système automatique d'analyse de texte basé sur des principes de rhétorique. L'analyse critique du discours a également eu des échos chez les étudiants de littérature (Gee 2005). Dans les départements de communication d'Amérique du Nord, l'analyse du discours est souvent pratiquée non par les rhétoriciens, mais par les chercheurs en communication interpersonnelle ou organisationnelle, en discours des médias, ou en argumentation. Les ethnographes de la communication comme Philipsen (1992) et Carbaugh (2005) font de l'analyse du discours, de même que les chercheurs reliés à la tradition du «langage comme interaction sociale " tels que Tracy (2002) et Fitch (1998). Jacobs et Jackson (1982) ont développé une théorie de l'argumentation basée sur les principes de l'analyse conversationnelle, et l'analyse critique du discours est populaire dans les programmes de communication. Cependant, les chercheurs qui considèrent leurs travaux comme relevant de la critique ou de la théorie rhétoriques doivent encore prendre connaissance d'une série d'articles qui leur présentent l'analyse du discours et leur montrent son utilité pour répondre aux types de questions qu'ils posent. Les approches de l'analyse du discours présentées et illustrées dans Rhetoric in Detail ont pour but d'illustrer les nombreuses manières dont une attention minutieuse et rigoureuse portée au langage peut être féconde pour les rhétoriciens.

\section{BIBLIOGRAPHIE}

Asen, Robert. 2004. « A Discourse Theory of Citizenship », Quarterly Journal of Speech 90, 189-211

Bakhtin, Michael. 1986. Speech Genres and Other Late Essays, trad. Vern W. McGee, Emerson, Caryl \& Michael Holquist (éds) (Austin : University of Texas Press)

Argumentation et Analyse du Discours, 9 | 2012 
Barton, Ellen \& Gail Stygall. 2002. Discourse Studies in composition (Cresskill, NJ : Hampton Press) Bazerman, Charles \& Paul Prior (éds). 2004. What writing does and how it does it: An Introduction to analyzing texts and Textual Practices (Mahwah, NJ : Lawrence Erlbaum)

Becker, Alton. L. 1995. Beyond Translation : Essays towards a Modern Philology (Ann Arbor : University of Michigan Press)

Bitzer, Lloyd.1968. « The Rhetorical Situation », Philosophy and Rhetoric 1, 1-14

Burke, Kenneth. 1945. A Grammar of Motives (Berkeley : University of California Press)

Carbaugh, Donald. 2005. Cultures in Conversation (Mahwah, NJ : Lawrence Erlbaum)

Cooper, Charles \& Sidney Greenbaum (éds). 1986. Studying Writing : Linguistic Approaches. Vol. 1, Written Communication Annual (Beverly Hills, CA : Sage)

Eggins, Suzanne. 1994. An Introduction to Systemic Functional Linguistics (London \& New-York : Pinter)

Fairclough, Norman. 2003. Analyzing Discourse : Textual Analysis for Social Research (London : Routledge)

Fairclough, Norman. 1992. Discourse and Social Change (Cambridge, UK : Polity)

Fitch, Kristine. 1998. Speaking Rationally: Culture, Communication and Interpersonal Connections (London : Guilford Press)

Foucault, Michel. 1980. Power/Knowledge : Selected Interviews and Other Writings, 1972-1977 (New York : Pantheon)

Gee, James Paul, 2005. An Introduction to Discourse Analysis : Theory and Method (London : Routledge) Geertz, Clifford. 1983. Local Knowledge : Further Essays in Interpretive Anthropology (New York : Basic Books)

Gumperz, John \& Dell Hymes (éds). 1972. Directions in Sociolinguistics : The Ethnography of Communication (New York : Holt Rinehart Winston)

Habermas, Jürgen. 1989. The Structural Transformation of the Public Sphere: An Inquiry Into a Category of Bourgeois Society (Cambridge : Polity)

Halliday, Michael Alexander Kirkwood. 1994. An Introduction to Functional Grammar (London :

Edward Arnold)

Handa, Carolyn. 2004. Visual Rhetoric in a Digital World : A Critical Sourcebook (Boston : Bedford/St. Martins)

Hauser, Gerard A. 1999. Vrenucalor Voices. The Rhetoric of Publics and Public Spheres (Columbia : University of South Carolina Press)

Hodge, Robert \& Gunther Kress. 1988. Social Semiotics (Cambridge, UK : Polity)

Jacob, Scott \& Sally Jackson. 1982. «Conversational Argument : A Discourse Analytic Approach », Cox J. Robert \& Charles A. Willard. Advances in Argumentation Theory and Research (Carbondale \& Edwardsville, IL : Southern Illinois University Press), 205-237

Johnstone, Barbara. 2008. Discourse Analysis (Oxford : Blackwell)

Kaufer, David \& Brian Butler. 2000. Designing Interactive Worlds with Words : Principles of Writing as Representational Composition (Mahwah, NJ : Lawrence Erlbaum) 
Kaufer, David Suguru Ishizaki, Brian Butler \& Jeff Collins. 2004. The Power of Words : Unveiling the Speaker and Writer's Hidden Craft (Mahwah, NJ : Lawrence Elbaum)

Labov, William. 1972. Sociolinguistics Patterns (Philadelphia : University of Pennsylvania Press)

Labov, William. 1963. « The social Motivation of a Sound Change », Word 19, 237-309

Leff, Michael. 2001. «Lincoln at Cooper Union : Neo-Classicism Revisited », Western Journal of Communication 65, 323-348

Levine, Philip \& Ron Scollon (éds). 2004. Discourse and Technology : Multimodal Discourses Analysis (Washington D.C. : Georgetown University Press)

McCormick, Christine B. (2003). « Metacognition and Learning », Reynolds, William M, Gloria E. Miller, \& Irving B. Weiner (éds.). Handbook of Psychology Vol. 7, « Educational Psychology » (New York : Wiley), 79-102

Miller, Carolyn. 1984. « Genre as Social Action », Quarterly Journal of Speech, 89 (2), 109-131

Pêcheux, Michel. 1969. Analyse automatique du discours (Paris : Dunod)

Philipsen, Gerry. 1992. Speaking Culturally: Explorations in Social Communication (Albany, N.Y : State University of New York Press)

Pike, Kenneth. 1967. Language in Relation to a Unified Theory of the Structure of Human Behavior (The Hague : Mouton)

Prelly, Lawrence. 2006. Rhetorics of Display (Columbia : University of South Carolina Press)

Raskin, Victor \& Irwin Weiser (éds). 1987. Language and Writing: Applications of Linguistics to Rhetoric and Composition (Norwood, N.J. : Ablex)

Sapir, Edward. 1949. Selected Writings of Edward Sapir in Language, Culture and Personality (Berkeley : University of California Press)

Sacks, Harvey, Emanuel Schlegoff \& Gail Jefferson. 1974. « A Simplest Systematics for the Organization of Turntaking for Conversation », Language 50, 696-735

Saussure, Ferdinand. 1916. Cours de linguistique générale (Paris : Payot)

Scollon, Ron \& Suzie Scollon. 2003. Discourses in Place : Language in the Material World (London : Routledge)

Swales, John. 1990. Genre Analysis : English in Academic and Research Settings (Cambridge :

Cambridge University Press)

Ten Have, Paul. 1999. Doing Conversation Analysis : A Practical Guide (London : Sage Publications)

Tracy, Keren. 2002. Everyday Talk: Building and Reflecting Identities (London : Guilford)

Vatz, Richard E. 1973. « The Myth of the Rhetorical Situation », Philosophy and Rhetoric 6 (3), 154-161

Wodak, Ruth. 1996. Disorders of Discourse (London : Longman)

\section{RÉSUMÉS}

Ce texte est une introduction à un ouvrage intitulé La rhétorique en détail, qui se propose de montrer l'importance de l'analyse du discours pour les études de rhétorique telles qu'elles sont aujourd'hui pratiquées aux Etats-Unis. Il insiste sur la façon dont une attention soutenue à la 
matérialité du langage peut enrichir les diverses branches de la rhétorique contemporaine en présentant une méthode fondée sur l'étude concrète des textes qui vise à dégager leur logique interne. Il offre ce faisant une définition de l'analyse du discours, une réflexion sur la question de l'agentivité, et une " heuristique » dans le sens d'un « un ensemble de procédures de découverte à appliquer méthodiquement ».

This text is the introduction to a book entitled Rhetoric in Detail that is intended to demonstrate the importance of Discourse Analysis for American Rhetorical Studies. It emphasizes the contribution that close attention to language can make to various branches of contemporary rhetoric, and offers an empirical approach based on the study of actual texts and talk, looking for their internal logic. It provides a definition of Discourse Analysis as well as an approach to the question of agency, and a heuristic in the sense of a "set of discovery procedures for systematic application"

INDEX

Mots-clés : agentivité, analyse du discours, heuristique, rhétorique

Keywords : agency, discourse analysis, heuristic, rhetoric

\section{AUTEURS}

\section{CHRISTOPHER EISENHART}

University of Massachusetts at Dartmouth

\section{BARBARA JOHNSTONE}

Carnegie Mellon University 